BULLETIN (New Series) OF THE

AMERICAN MATHEMATICAL SOCIETY

Volume 51, Number 2, April 2014, Page 335

S 0273-0979(2014)01451-X

Article electronically published on January 10, 2014

\title{
A NOTE ON THE BULLETIN COVER
}

\author{
MARSHALL SLEMROD
}

Most of readers of the Bulletin of the American Mathematical Society are familiar with the well-advertised Clay Millennium Prize problems. One of the problems on the list is prove or disprove global in time existence and uniqueness of smooth solutions for the initial value problem for the Navier-Stokes equations in three space dimensions. One version is posed in all three dimensional space and the other is restricted to the case of periodic boundary conditions. Since this is a problem arising in continuum mechanics, one may ask what is the status of a reductionist approach to derivation of both the Navier-Stokes equations and the related inviscid Euler equations as a limit of "atomistic" theory.

Indeed this seems to have been what Hilbert was proposing as part of his 6th problem in his famous 1900 ICM-Paris address. In particular Hilbert mentioned the Boltzmann equation as an appropriate axiomatic starting point though he was not, of course, the first to observe the connection between the atomistic Boltzmann formulation of gas dynamics and equations of continuum mechanics. In this regard may be noted the work of Maxwell who provided both a derivation of the steady inviscid Euler equations and the first case where the Boltzmann equation is used to derive the equations of gas dynamics beyond the Navier-Stokes Fourier theory [J. C. Maxwell, On the stresses in rarefied gases arising from inequalities of temperature, Philosophical Transactions of the Royal Society, 170 (1879), 231-256].

Following Maxwell, we have chronologically Hilbert's 1900 address, Hilbert's 1912 paper in which he presented his own way to approximate solutions of the Boltzmann equation as a series expansion, and the independently written papers of Chapman and Enskog, both done in the period 1916-1917. As noted by C. Cercignani in his biography of Boltzmann [C. Cercignani, Ludwig Boltzmann, The Man Who Trusted Atoms, Oxford University Press, Oxford, 1998], Enskog's approach to the problem (based on Hilbert's 1912 idea) was then adopted by Chapman and Cowling in their book, The Mathematical Theory of Non-Uniform Gases (Cambridge University Press, Cambridge, 1939), and thus became known as the Chapman-Enskog method. It is the mathematical analysis of this method that intrigues mathematicians to this day and is a worthy topic of the survey articles, one by Gorban and Karlin and the other by St. Raymond, that appear in this volume of the Bulletin.

University of Wisconsin, Madison, Madison, Wisconsin 53706

E-mail address: slemrod@math.wisc.edu 